\title{
Estrogens, Conjugated
}

National Cancer Institute

\section{Source}

National Cancer Institute. Estrogens, Conjugated. NCI Thesaurus. Code C482.

Purified orally bioavailable female sex hormones isolated from pregnant mare urine or synthetically derived from plant materials and primarily conjug ated as sulfate esters. Estrogen binds to and activates specific nuclear receptors, which, in turn, bind to estrogen response elements (EREs) in target genes, resulting in histone acetylation, alteration of chromatin conformation, and initiation of transcription. ( $\mathrm{NCl04)}$ 